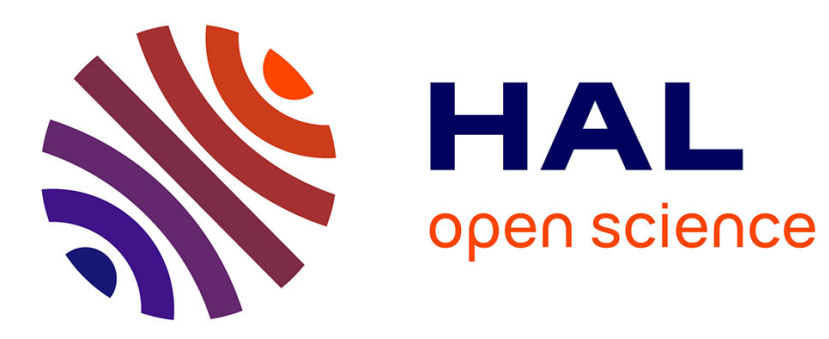

\title{
Formation de l'interface métal/InP et de diodes Schottky sur InP
}

\author{
A. Ismail, J.-M. Palau, L. Lassabatère
}

\section{To cite this version:}

A. Ismail, J.-M. Palau, L. Lassabatère. Formation de l'interface métal/InP et de diodes Schottky sur InP. Revue de Physique Appliquée, 1984, 19 (3), pp.205-214. 10.1051/rphysap:01984001903020500 . jpa-00245184

\section{HAL Id: jpa-00245184 https://hal.science/jpa-00245184}

Submitted on 1 Jan 1984

HAL is a multi-disciplinary open access archive for the deposit and dissemination of scientific research documents, whether they are published or not. The documents may come from teaching and research institutions in France or abroad, or from public or private research centers.
L'archive ouverte pluridisciplinaire HAL, est destinée au dépôt et à la diffusion de documents scientifiques de niveau recherche, publiés ou non, émanant des établissements d'enseignement et de recherche français ou étrangers, des laboratoires publics ou privés. 
Classification

Physics Abstracts

73. 30Y

\title{
Formation de l'interface métal/InP et de diodes Schottky sur InP
}

\author{
A. Ismail, J. M. Palau et L. Lassabatère \\ Laboratoire d'études des surfaces, interfaces et composants $\left({ }^{*}\right)$, \\ U.S.T.L., place Eugène Bataillon, 34060 Montpellier Cedex, France
}

(Reçu le 25 juillet 1983, révisé le 2 novembre, accepté le 4 novembre 1983)

\begin{abstract}
Résumé. - On étudie principalement par la méthode Kelvin mais aussi par spectrométrie Auger les surfaces (100) chimiques et (110) clivées de InP, l'interaction de cette dernière avec un métal réactif ( $\mathrm{Al}$ ) ou non réactif $(\mathrm{Au}, \mathrm{Ag})$. On compare ensuite les résultats fournis par l'étude des surfaces aux hauteurs de barrières mesurées dans les diodes réalisées sur les deux types de surface. On montre que InP se caractérise par un ancrage du niveau de Fermi situé dans la moitié supérieure de la bande interdite. Cet ancrage se produit : dans le cas des surfaces libres (100) ou (110) avec défauts de clivage, après dépôt d'une fraction de monocouche de métal, dans les diodes. On propose comme explication la présence d'états donneurs et accepteurs associés à des défauts du semiconducteur dont les densités relatives seraient liées aux détails de la formation de l'interface.
\end{abstract}

\begin{abstract}
A.E.S. and mainly Kelvin method are used to study chemical (100) and cleaved (110) InP surfaces and their interaction with reactive $(\mathrm{Al})$ or unreactive $(\mathrm{Au}, \mathrm{Ag})$ metals. The surface barrier is compared to the barrier height measured in the diode. Results show that InP is characterized by a surface Fermi level pinning in the upper half part of the gap. This pinning occurs : for the free chemical (100) faces and for the cleaved faces presenting cleavage defects, after deposition of a submonolayer metal quantity, in the diodes. We propose that pinning results from acceptor and donor states whose relative densities are depending upon the details of the formation of the interface. States are supposed to be due to defects localized at the semiconductor surface.
\end{abstract}

\section{Introduction.}

Les concepts de base utilisés encore aujourd'hui pour décrire le contact métal semiconducteur ont principalement pour origine les travaux de Mott et Schottky [1-3], de Bethe [4] puis de Bardeen [5] et Cowley et Sze [6]. Si les théories décrivant les mécanismes de transport du courant [7] expliquent de façon plutôt satisfaisante les observations expérimentales, la question de l'origine exacte de la barrière de Schottky n'est toujours pas véritablement tranchée. Les approches fondamentales visant à décrire les caractéristiques du contact par l'interpénétration des fonctions d'ondes du métal et du semiconducteur [8-12] ont été remises en question par les résultats expérimentaux fournis par les techniques sophistiquées d'analyse des surfaces appliquées en particulier à l'étude des III-V et singulièrement à GaAs [13].

Différentes approches se sont alors développées. Spicer et collaborateurs [14-16] proposent un modèle dans lequel l'interaction du métal, plus généralement de l'adatome, avec le semiconducteur produit, à la surface ou au voisinage de la surface de celui-ci, des

(*) U.R. No 1022 associée au C.N.R.S. défauts, principalement des lacunes de l'anion ou du cation. Ces défauts correspondraient à des états discrets, situés dans la bande interdite et qui, comme dans le modèle de Bardeen, ancreraient le niveau de Fermi à l'interface. L'idée d'un ancrage du niveau de Fermi par des états liés à des défauts est aussi soutenue par Williams et collaborateurs $[17,18]$ qui soulignent cependant des différences dans la position de l'ancrage corrélées à la réactivité chimique du métal avec le semiconducteur. L'effet de la réactivité du métal et de la chaleur de réaction sur la hauteur de la barrière et en même temps sur les phénomènes d'interdiffusion à l'interface est particulièrement mis en évidence par Brillson et collaborateurs [13, 19-22] qui montrent d'une part que le comportement redresseur est sensiblement modifié par l'interposition d'un petit nombre de monocouches d'un métal de réactivité différente, d'autre part que la largeur de l'interface est plus faible si le métal est réactif. Pour Freeouf et Woodall [23-25] la diffusion du cation dans le métal et la formation d'îlots riches en anions conduit à la présence de différentes phases ayant chacune son propre travail de sortie. Il en résulte un travail de sortie "effectif" moyen à prendre en compte pour déterminer la hauteur de barrière par la simple théorie de Schottky. 
Toutes ces approches tendent donc à expliquer le comportement du contact Schottky par différentes imperfections par rapport au contact abrupt théorique. Une interprétation différente est soutenue par Sebenne et collaborateurs [26] qui envisagent qu'une grande partie des états responsables de l'ancrage du niveau de Fermi à l'interface provient d'un retour dans la bande interdite des états intrinsèques qui avaient été repoussés dans les bandes par la relaxation des atomes de surface. Ce modèle, qui considère donc une modification de la relaxation induite par les liaisons des atomes du semiconducteur avec ceux du métal, implique implicitement une interface abrupte.

Si depuis une dizaine d'années beaucoup d'études ont été effectuées sur GaAs, beaucoup moins de résultats sont disponibles pour InP. Ces résultats [27-36] font cependant apparaître que si la plage des valeurs possibles pour la position du niveau de Fermi à l'interface est caractéristique du semiconducteur, des hauteurs de barrière sensiblement différentes sont produites par les métaux réactifs et non réactifs. Peu d'expérimentateurs ont étudiés le contact Schottky à la fois sur les surfaces (110) obtenues par clivage et (100) obtenues par nettoyage chimique, d'une part par des techniques d'analyse de surface, d'autre part par une caractérisation électrique des diodes. Nous présentons ici une étude réalisée dans ce sens à la fois sur InP types $\mathrm{n}$ et $\mathrm{p}$ avec comme métaux l'argent, l'or et l'aluminium. Les surfaces sont caractérisées par des mesures topographiques du travail de sortie et du photovoltage utilisant la technique du condensateur vibrant de Kelvin et par spectrométrie Auger.

L'ancrage du niveau de Fermi à la surface, qui doit se traduire par un rapprochement des travaux de sortie des échantillons de types $n$ et $p$, peut donc être mis en évidence de façon simple dans notre dispositif. De plus, le photovoltage, dont la valeur dépend de la barrière de potentiel de surface, fournit des informations quantitatives sur cette dernière. Les diodes sont étudiées de façon classique à la température ambiante par $I(V)$ en direct et $C(V)$ en inverse.

\section{Dispositif expérimental.}

L'ensemble expérimental déjà décrit dans d'autres publications $[37,38]$ est constitué du bâti à ultra vide $\left(10^{-10}\right.$ torr) et de ses équipements : sonde de Kelvin, spectromètre Auger à miroir cylindrique, spectromètre de masse, cellules d'évaporation. Le volume dans lequel est confinée l'évaporation du métal est limitée par des panneaux cryogéniques. La vitesse d'évaporation minimale contrôlée par le spectromètre de masse est de l'ordre d'une monocouche à la minute. La sonde de Kelvin a une résolution spatiale de l'ordre de $250 \mu \mathrm{m}$ et une sensibilité d'environ $1 \mathrm{meV}$. La distance sonde échantillon est maintenue constante (typiquement $30 \pm 1 \mu \mathrm{m})$ par un asservissement et permet une étude topographique de la différence de potentiel de contact

$$
\Delta \phi=\phi_{\text {SC }}-\phi_{\text {reff. }}
$$

obtenue en déplaçant l'échantillon devant l'électrode de référence. Le porte échantillon reçoit en même temps deux échantillons $\mathrm{n}$ et $\mathrm{p}$, qui peuvent donc être comparés in situ, barreaux à cliver ou plaquettes nettoyées chimiquement. Les échantillons peuvent être éclairés par une lampe de $100 \mathrm{~W}$ à filament de tungstène focalisée par un miroir elliptique.

Le dopage des échantillons utilisés est le suivant : barreaux $n=5 \times 10^{15} \mathrm{~cm}^{-3}$ et $p=2,5 \times 10^{15} \mathrm{~cm}^{-3}$, plaquettes $n=5,5 \times 10^{15} \mathrm{~cm}^{-3}$ et $p=4,3 \times 10^{15} \mathrm{~cm}^{-3}$.

Ceci correspond à une position du niveau de Fermi en volume telle que $E_{\mathrm{Fp}}-E_{\mathrm{V}} \approx 230 \mathrm{meV}$ pour les types $\mathrm{p}$ et $E_{\mathrm{C}}-E_{\mathrm{Fn}} \approx 120 \mathrm{meV}$ pour les types $\mathrm{n}$.

Le nettoyage des plaquettes est effectué juste avant la mise sous vide à l'aide de la solution classique : $3 \mathrm{H}_{2} \mathrm{SO}_{4}, 1 \mathrm{H}_{2} \mathrm{O}_{2}, 1 \mathrm{H}_{2} \mathrm{O}$.

\section{Face (110) clivée.}

3. 1 SURFACE LIBRE. - L'étude topographique à l'aide de la sonde de Kelvin des surfaces clivées de InP montre que, comme dans le cas de GaAs [37], les variations du travail de sortie sont étroitement corrélées aux défauts de clivage. Les résultats détaillés de cette étude, qui feront l'objet d'une autre publication, peuvent être résumés comme suit. Les défauts de clivage tendent à rapprocher les travaux de sortie des types $\mathrm{n}$ et $\mathrm{p}$ et produisent dans les deux cas une courbure des bandes en surface dans le sens de l'appauvrissement ou de l'inversion (photovoltage négatif sur type $n$ et positif sur type $p$ ). Ces résultats conduisent, comme dans le cas de GaAs, à associer des états accepteurs et donneurs aux défauts de clivage.

La bonne corrélation entre les valeurs du photovoltage et les variations du travail de sortie montrent que ces dernières sont essentiellement imputables à la courbure des bandes. On peut donc connaître en tout point la valeur du potentiel de surface $V_{\mathrm{S}}$ ainsi que la différence de potentiel de contact $\Delta \phi_{0}$ en bande plate. De plus on vérifie que la différence $\Delta \phi_{0 \mathrm{p}}-\Delta \phi_{0 \mathrm{n}}$ est égale à la valeur théorique $\phi_{0 \mathrm{p}}-\phi_{0 \mathrm{n}}=E_{\mathrm{Fn}}-E_{\mathrm{Fp}}$ calculée à partir du dopage. Les valeurs extrêmes de $V_{\mathrm{Sn}}$ et $V_{\mathrm{Sp}}$ que nous avons observées montrent que lorsque la densité des défauts de clivage croît, le niveau de Fermi en surface tend à être ancré à un niveau d'énergie que l'on peut situer entre 0,3 et $0,5 \mathrm{eV}$ en dessous du bas de la bande de conduction.

\subsection{EFFET DU DÉPÔT D'UNE FAIBLE QUANTITÉ DE MÉTAL.} Le dépôt d'une fraction de monocouche de métal a un effet considérable sur les surfaces clivées : les nonuniformités du travail de sortie dues aux défauts de clivage ont pratiquement disparu, le travail de sortie du type $\mathrm{n}$ et du type $\mathrm{p}$ sont presque égaux, la variation du travail de sortie, qui dépend du métal utilisé, est particulièrement importante sur le type $p$ pour lequel $\Delta \phi$ diminue (Fig. 1).

Les variations du travail de sortie prises en prenant comme référence le travail de sortie $\phi_{0 \mathrm{n}}$ du type $\mathrm{n}$ en bandes plates sont indiquées figure 2. Dans le tableau I sont reportées les variations du travail de 

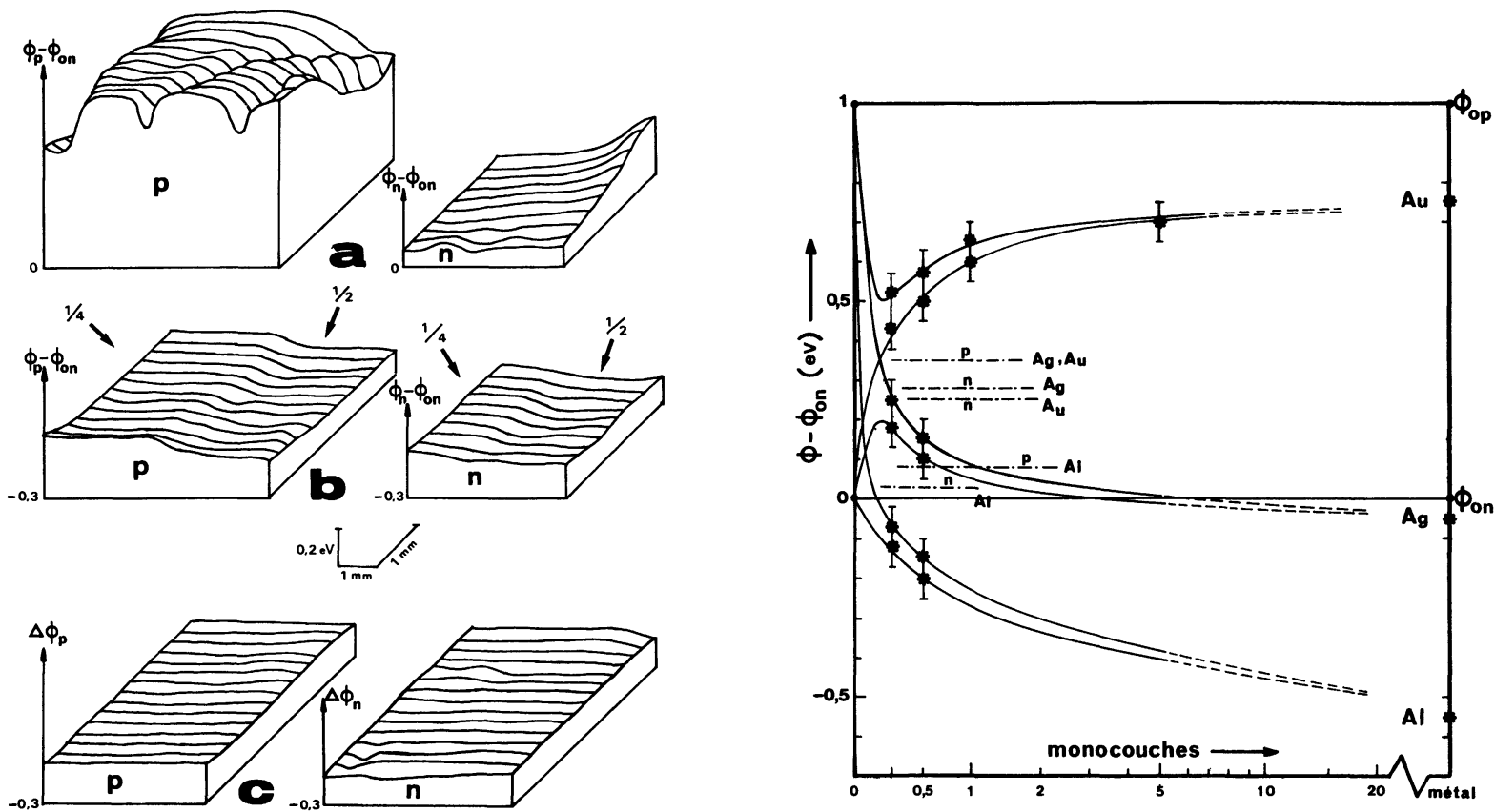

Fig. 1. - a - Exemples de topographies du travail de sortie de faces (110) présentant des défauts de clivages. Les travaux de sortie du type pet du type $n$ sont portés en prenant comme origine le travail de sortie $\phi_{0 n}$ du type $n$ en bande plate. $b$ - Modification des topographies précédentes après dépôt de $1 / 4$ et $1 / 2$ monocouche d'aluminium sur deux moitiés de chaque échantillon. c - Exemples de topographies de la différence de potentiel de surfaces (100) d'un échantillon de type $\mathrm{p}$ et d'un échantillon de type $\mathrm{n}$.

[a - Examples of work function topographies of (110) faces presenting some cleavage defects. Work function for both $\mathrm{n}$ and $\mathrm{p}$ types is origined with respect to the work function $\phi_{0 n}$ of the $n$ type in flat band condition. $b-$ Modification of the previous topographies by $1 / 4$ and $1 / 2$ monolayer of aluminium deposited on two half parts of each sample. c - Examples of CPD topographies of the (100) etched face of $n$ and $p$ type samples.]

Fig. 2. - Valeurs, par rapport au type $\mathrm{n}$ en bande plate, du travail de sortie des surfaces (110) clivées recouvertes de différentes quantités d'or, d'argent ou d'aluminium. Les valeurs obtenues sur couches massives sont aussi indiquées. Les niveaux représentés en pointillés indiquent quels seraient les travaux de sortie après dépôt de 0,25 à 0,5 monocouche si l'on tenait seulement compte de la courbure des bandes.

[Work function values, with respect to the $\mathrm{n}$ type in flat band condition, of cleaved (110) surfaces for different coverages of gold, silver or aluminium. Are also plotted work function values of thick layers of these metals. Dotted levels indicate work function corresponding to $1 / 4$ and $1 / 2$ monolayer that would be obtained if only band bending were considered.]

Tableau I. - Travail de sortie et photovoltage des faces (110) clivées après dépôt d'une fraction de monocouche de métal.

[Work function and SPV of the cleaved surfaces after deposition of metal (1/4 and 1/2 monolayer).]

\begin{tabular}{|l|c|c|c|c|c|}
\cline { 2 - 6 } \multicolumn{1}{c|}{} & $\phi_{\mathrm{p}}-\phi_{0 \mathrm{p}}(\mathrm{meV})$ & $\phi_{\mathrm{n}}-\phi_{0 \mathrm{n}}(\mathrm{meV})$ & $\phi_{\mathrm{p}}-\phi_{\mathrm{n}}(\mathrm{meV})$ & $S P V_{\mathrm{p}}(\mathrm{mV})$ & $S P V_{\mathrm{n}}(\mathrm{mV})$ \\
\hline $0,25 \mathrm{mc} \mathrm{Ag}$ & $-750 \pm 50$ & $180 \pm 50$ & $70 \pm 20$ & $260 \pm 10$ & $-60 \pm 5$ \\
\hline $0,5 \mathrm{mc} \mathrm{Ag}$ & $-850 \pm 50$ & $100 \pm 50$ & $50 \pm 20$ & $280 \pm 10$ & $-80 \pm 5$ \\
\hline $0,25 \mathrm{mc} \mathrm{Au}$ & $-470 \pm 50$ & $430 \pm 50$ & $100 \pm 20$ & $200 \pm 10$ & $-50 \pm 5$ \\
\hline $0,5 \mathrm{mc} \mathrm{Au}$ & $-430 \pm 50$ & $500 \pm 50$ & $70 \pm 20$ & $230 \pm 10$ & $-70 \pm 5$ \\
\hline $0,25 \mathrm{mc} \mathrm{Al}$ & $-1070 \pm 50$ & $-120 \pm 50$ & $50 \pm 20$ & $500 \pm 20$ & $-5 \pm 2$ \\
\hline $0,5 \mathrm{mc} \mathrm{Al}$ & $-1150 \pm 50$ & $-200 \pm 50$ & $50 \pm 20$ & $500 \pm 20$ & $-5 \pm 2$ \\
\hline
\end{tabular}


sortie et la valeur du photovoltage après dépôt de 0,25 et 0,5 monocouche. Rappelons que les valeurs de $\phi_{\mathrm{p}}-\phi_{\mathrm{n}}$ sont mesurées directement entre les deux échantillons et de ce fait sont connues avec une meilleure précision que $\phi_{\mathrm{p}}-\phi_{0 \mathrm{p}}$ ou $\phi_{\mathrm{n}}-\phi_{0 \mathrm{n}}$. Le photovoltage est très uniforme même quand la surface initiale présentait de très importantes variations du travail de sortie et donc du photovoltage. Il évolue peu pour des épaisseurs métalliques qui restent inférieures ou égales à la monocouche. Le signe du photovoltage indique que les types $n$ et $p$ présentent dans tous les cas une barrière d'appauvrissement (voire d'inversion) en surface, et il apparaît très clairement que dans le cas de l'aluminium cette barrière de surface est plus grande que pour l'or et l'argent dans le cas du type $p$, plus faible dans le cas du type $n$. Dans tous les cas cependant le photovoltage indique que la barrière sur type $p$ est plus grande que celle sur type $n$. Il semble que la majeure partie de la décroissance du travail de sortie du type $p$ puisse être interprétée par la variation de la barrière de surface $q V_{\mathrm{s}}$. En effet le travail de sortie $\phi$, qui s'exprime par la relation : $\phi=\chi+E_{\mathrm{g}}-\left(E_{\mathrm{F}}-E_{\mathrm{V}}\right)-q V_{\mathrm{S}}$, doit diminuer pour le type $\mathrm{p}$ s'il apparaît une barrière d'appauvrissement $\left(V_{\mathrm{S}}>0\right.$, ce qui correspond au photovoltage observé). Mais une partie des variations de $\phi$ peut être due à une variation de l'affinité électronique $\chi$. Dans le cas de l'aluminium, il est clair qu'une telle variation existe. En effet, $\phi_{\mathrm{p}}-\phi_{0 \mathrm{p}}=$ $-1,15 \mathrm{eV}$ et $\phi_{\mathrm{n}}-\phi_{0 \mathrm{n}}=-0,2 \mathrm{eV}$ pour 0,5 monocouche. Compte tenu du dopage des échantillons $E_{\mathrm{Fp}}-E_{\mathrm{F}}=0,23 \mathrm{eV}$ et $E_{\mathrm{Fn}}-E_{\mathrm{V}}=1,23 \mathrm{eV} . \mathrm{Si} \chi$ était constante, le niveau de Fermi en surface serait dans la bande de conduction et le type n, en accumulation, donnerait un photovoltage positif ce qui est contraire à l'expérience. On doit donc considérer de façon générale que le dépôt même d'une faible quantité de métal modifie l'affinité électronique en même temps qu'il produit ou modifie la courbure des bandes. On peut raisonnablement supposer que la variation d'affinité électronique $\Delta \chi$ est indépendante du dopage. Alors

$$
\begin{aligned}
\phi_{\mathrm{p}}-\phi_{\mathrm{n}}=E_{\mathrm{Fn}}-E_{\mathrm{Fp}}-q\left(V_{\mathrm{Sp}}-V_{\mathrm{Sn}}\right) & = \\
& =E_{\mathrm{FSn}}-E_{\mathrm{FSp}} .
\end{aligned}
$$

Nous trouvons pour les trois métaux que, même pour 0,25 monocouche, $\phi_{\mathrm{p}}-\phi_{\mathrm{n}}<100 \mathrm{mV}$. La position du niveau de Fermi en surface du type $n$ et celle du type $p$ sont très proches, l'ancrage s'effectue presque au même niveau.

$$
V_{\mathrm{Sp}}-V_{\mathrm{Sn}}=\frac{E_{\mathrm{Fn}}-E_{\mathrm{Fp}}-\left(\phi_{\mathrm{p}}-\phi_{\mathrm{n}}\right)}{q}
$$

soit, compte tenu des dopages, des signes des potentiels de surface et des valeurs de $\phi_{\mathrm{p}}-\phi_{\mathrm{n}}: V_{\mathrm{sp}}+$ $\left|V_{\mathrm{Sn}}\right| \approx 0,95 \mathrm{~V}$. Il est clair que les valeurs du photovoltage, qui pourtant sont des valeurs largement saturées c'est-à-dire qui n'augmentent plus avec l'intensité lumineuse, ne satisfont pas à cette condition. Ceci montre que, contrairement aux propositions de Brillson [39, 40], le photovoltage n'est pas égal à $V_{\mathrm{s}}$. Dans le cas des surfaces libres où $V_{\mathrm{s}}$ est connu, nous avons pu tracer les courbes donnant le photovoltage en fonction de $V_{\mathrm{s}}$. Nous supposons, au moins en première approximation, que ces courbes sont encore utilisables, c'est-à-dire que, dans le cas du dépôt métallique, elles sont atténuées dans un rapport constant qui dépend de la nature du métal et de l'épaisseur de la couche mais qui peut être calculé puisque $V_{\mathrm{Sp}}-V_{\mathrm{Sn}}$ est connue. On peut alors déduire des valeurs du photovoltage les valeurs de $V_{\mathrm{S}}$ portées dans le tableau II. Ces valeurs de $V_{\mathrm{s}}$, en accord satisfaisant avec les résultats de photoémission publiés [27-29, 33-36], montrent que l'ancrage du niveau de Fermi en surface s'effectue dans la moitié supérieure de la bande interdite à environ $400 \mathrm{meV}$ du bas de la bande de conduction dans le cas de l'or et de l'argent, à environ $150 / 200 \mathrm{meV}$ dans le cas de l'aluminium. Il est à remarquer que l'ancrage se produit dans la même plage d'énergie que celle vers laquelle tend à être ancré le niveau de Fermi en surface sous l'effet d'une forte densité d'états de clivage. Les valeurs de $V_{\mathrm{S}}$ du tableau II permettent de déduire la variation d'affinité électronique $\Delta \chi$ par : $\phi-\phi_{0}=\Delta \chi-q V_{\mathrm{s}}$. Les valeurs de $\Delta \chi$ ainsi calculées sont reportées dans le tableau III dans lequel on a aussi indiqué les valeurs,

Tableau II. - Potentiel de surface des faces (110) clivées après dépôt de 0,25 à 0,5 monocouche de métal.

[Surface potential of the (110) cleaved surfaces after deposition of $1 / 4$ or $1 / 2$ monolayer of metal.]

\begin{tabular}{|c|r|r|c|}
\cline { 2 - 4 } \multicolumn{1}{c|}{} & \multicolumn{1}{c|}{$\mathrm{Ag}$} & \multicolumn{1}{c|}{$\mathrm{Au}$} & \multicolumn{1}{c|}{$\mathrm{Al}$} \\
\hline$V_{\mathrm{Sn}}(\mathrm{mV})$ & $-280 \pm 50$ & $-250 \pm 50$ & $-30 \pm 20$ \\
\hline$V_{\mathrm{Sp}_{\mathrm{p}}}(\mathrm{mV})$ & $650 \pm 50$ & $650 \pm 50$ & $920 \pm 50$ \\
\hline
\end{tabular}

Tableau III. - Variation de l'affinité électronique après dépôt de 0,25 et 0,5 monocouche de métal et travail de sortie d'une couche de métal massive (par rapport au type $\mathrm{n}$ en bande plate).

[Electron affinity variations due to $1 / 4$ and $1 / 2$ monolayer and work function of a thick layer of the metal (origin is $\mathbf{n}$ type in flat band).]

\begin{tabular}{|c|r|r|c|}
\hline$(\mathrm{meV})$ & \multicolumn{1}{c|}{$\mathrm{Ag}$} & \multicolumn{1}{c|}{$\mathrm{Au}$} & $\mathrm{Al}$ \\
\hline$\Delta \chi(0,25 \mathrm{mC})$ & -100 & 180 & -150 \\
\hline$\Delta \chi(0,5 \mathrm{mC})$ & -190 & 240 & -230 \\
\hline$\phi_{\text {metal }}-\phi_{0 \mathrm{n}}$ & -50 & +750 & -550 \\
\hline
\end{tabular}


par rapport au type $\mathrm{n}$ en bande plate, du travail de sortie du métal. Les valeurs de $\Delta \chi$ bien qu'entachées d'une incertitude importante montrent clairement qu'elles contribuent à rapprocher le travail de sortie du semiconducteur, compte tenu de sa courbure de bande, de celui du métal. Comme dans le cas de GaAs [41, 42], le dépôt d'une faible quantité de métal sur la surface clivée (110) de InP a un double effet. Premièrement, il produit un ancrage du niveau de Fermi en surface dont la cause, compte tenu du fait qu'il apparaît pour les quantités de métal bien inférieure à la monocouche, ne peut être que la création d'états de surface accepteurs et donneurs. Les caractéristiques énergétiques de ces états sont vraisemblablement voisines de celles des états correspondant aux défauts de clivage qui conduisent aussi à un ancrage dans la même région de la bande interdite. Cependant, une modulation des caractéristiques de ces états par la nature du métal peut être envisagée (par exemple, variation de la densité des donneurs par rapport aux accepteurs).

Deuxièmement, le dépôt d'une faible quantité de métal conduit aussi à une modification de l'affinité électronique du semiconducteur par création d'un dipôle de surface. L'origine de ce dipôle qui peut être véritablement microscopique, dans le cas d'une interface abrupte ou semimacroscopique, c'est-à-dire étalé sur plusieurs couches atomiques, reste à préciser. Pour des raisons techniques et en particulier parce que le travail de sortie est modifié par l'impact d'un faisceau d'électrons [43, 44], nous n'avons pas effectué une étude systématique en spectroscopie Auger de l'effet de l'épaisseur de métal déposé. Il ressort cependant de nos observations que, pour les trois métaux, que nous avons utilisés, c'est le pic du phosphore qui est atténué le premier. Dans le cas de l'or et de l'aluminium, il est clair qu'une importante interdiffusion a lieu. En effet, l'indium est nettement visible à la surface d'une couche d'aluminium de $5000 \AA$ et des traces d'indium et de phosphore sont visibles à la surface d'une couche de $1500 \AA$ d'or. Dans ces conditions, l'interprétation de $\Delta \chi$ par un vrai dipôle est peu vraisemblable au-delà d'une monocouche et l'idée d'une modification du travail de sortie (et donc de $\chi$ ) par l'apparition de matériaux composites proposée par Freeouf [23-25] est plus séduisante et plus en accord avec le fait que la valeur de $\Delta \chi$ assure, en quelque sorte, un passage progressif au travail de sortie du métal.

3.3 ETUDE DES DIODES. - Les diodes sont obtenues par évaporation du métal au travers d'un masque installé sur la cheminée d'évaporation. Les premières couches sont déposées à la même vitesse que pour les études précédentes, soit une à deux monocouches par minute. Elles sont ensuite caractérisées à l'extérieur du bâti et à la température ambiante par des études $I(V)$ en direct et $C(V)$ en inverse, dont l'exploitation est effectuée de façon classique par les relations :

$$
\begin{gathered}
I=A^{*} S T^{2} \exp \left(-\frac{\phi_{\mathrm{B}}}{k T}\right)\left(\exp \frac{q V}{n k T}-1\right) \\
1 / C^{2}=\frac{2}{S^{2} q \varepsilon d}\left(V_{\mathrm{D}}-V-\frac{k T}{q}\right)
\end{gathered}
$$

où $d$ est le dopage, $A_{\mathrm{p}}^{*}=84$ et $A_{\mathrm{n}}=9,36 \mathrm{~A} \mathrm{~cm}^{-2} \mathrm{~K}^{-2}$.

Les valeurs de $\phi_{\mathrm{B}}$ et de la barrière $\phi_{\mathrm{BC}}$ correspondant à $V_{\mathrm{D}}$ sont données dans le tableau IV. Globalement, ces hauteurs de barrières sont en accord avec les valeurs de $V_{\mathrm{s}}$ correspondant à une fraction de monocouche et, compte tenu des erreurs expérimentales, on peut considérer que, en première approximation, les valeurs des barrières de surface et dans les diodes sont comparables. En particulier, le décalage du niveau de Fermi dans le cas de l'aluminium, par rapport à l'or et à l'argent, est conservé pour la couche massive. Cependant il est clair que certains désaccords existent d'une part entre résultats sur les diodes elles-mêmes entre les barrières obtenues par $I(V)$ et $C(V)$, d'autre part entre barrières de surface et barrières dans les diodes.

Pour l'argent, l'écart entre $\phi_{\mathrm{Bp}}$ et $\phi_{\mathrm{BCp}}$, qui n'est que de $70 \mathrm{meV}$, est suffisamment faible pour être expliqué par les mécanismes usuels d'abaissement de barrière (effet de force image, émission thermoionique assistée par le champ). On peut admettre comme hauteurs de barrière des types n et $\mathrm{p}: \phi_{\mathrm{Bp}}=850 \mathrm{meV}$ et $\phi_{\mathrm{Bn}}=500 \mathrm{meV}$ soit $\phi_{\mathrm{Bn}}+\phi_{\mathrm{Bp}}=E_{\mathrm{g}}$. Ces valeurs, comparées à $V_{\mathrm{Sn}}$ et $V_{\mathrm{Sp}}$ du tableau II, montrent que la barrière finale est pratiquement établie pour moins d'une monocouche. Nos conclusions concernant la position et l'évolution de l'ancrage sont en accord avec les résultats publiés par Brillson et al. [33] et par Mc Kinley et al. [27].

Dans le cas de l'aluminium, l'écart entre $\phi_{\mathrm{Bp}}$ et $\phi_{\mathrm{BC}}$ est considérable et la valeur de $\phi_{\mathrm{BCp}}$, qui correspondrait à un niveau de Fermi de surface située dans la

Tableau IV. - Hauteur de barrières et facteurs d'idélité des diodes réalisées sur surfaces (110) clivées.

[Barrier hight and ideality factor for the diodes achieved on cleaved surfaces.]

\begin{tabular}{|c|c|c|c|c|}
\cline { 2 - 5 } \multicolumn{1}{c|}{} & $\mathrm{Ag}$ & $\mathrm{Au}$ & $\mathrm{Al}$ \\
\hline \multirow{4}{*}{ type $\mathrm{p}$} & $\phi_{\mathrm{B}}(\mathrm{meV})$ & 780 & 800 & 1000 \\
\cline { 2 - 5 } & $n$ & 1,10 & 1,40 & 1,15 \\
\cline { 2 - 5 } & $V_{\mathrm{D}}(\mathrm{mV})$ & 620 & 760 & 1150 \\
\cline { 2 - 5 } & $\phi_{\mathrm{Bc}}(\mathrm{meV})$ & 850 & 990 & 1370 \\
\hline \multirow{3}{*}{ type n } & $\phi_{\mathrm{B}}(\mathrm{meV})$ & 515 & 430 & \multirow{2}{*}{ ohmique } \\
\cline { 2 - 5 } & $n$ & 1,20 & 1,02 & \\
\hline
\end{tabular}


bande de conduction, n'est pas très réaliste. On doit envisager une modification importante de la forme du sommet de la barrière. En effet, les mesures de $C(V)$ en inverse font intervenir la limite interne de la zone de charge d'espace et la valeur de $\phi_{\mathrm{BC}}$ est en fait obtenue par extrapolation d'une zone de charge d'espace parabolique. Nous prendrons comme hauteur réelle de la barrière 1100 à $1150 \mathrm{meV}$ ce qui peut satisfaire à la fois à la valeur de $\phi_{\mathrm{Bp}}$ mesurée en $I(V)$, si l'on prend en compte les abaissements classiques possibles, et au fait que le contact est ohmique ou quasi ohmique sur type $\mathrm{n}$. L'ancrage du niveau de Fermi à l'interface se produirait donc dans les diodes à environ $200 \mathrm{meV}$ en dessous de la $\mathrm{BC}$. Il n'aurait donc pratiquement pas changé par rapport à sa position après dépôt de moins d'une monocouche. Les conclusions sont encore en assez bon accord avec les résultats de Brillson et al. [33] et de Mc Kinley et al. [28]. Dans le cas de l'or aussi, la différence entre $\phi_{\mathrm{BP}}$ et $\phi_{\mathrm{BCp}}$ est trop grande pour être expliquée par des processus classiques d'abaissement de barrière. Mais il faut noter la valeur particulièrement élevée du facteur d'idéalité qui est un indice sérieux d'une évolution de $\phi_{\mathrm{B}}$ avec la tension appliquée. Le type $\mathrm{n}$ semble être un indicateur plus fiable de la position de l'ancrage dans les diodes qui se situerait donc à environ $400-450 \mathrm{meV}$ en dessous de la BC, en accord satisfaisant avec nos mesures pour moins d'une monocouche. Dans le cas de l'or aussi, l'ancrage final serait établi très tôt, en accord avec les résultats de Spicer et al. $[34,35]$ mais en désaccord avec les résultats de Brillson et al. [33] qui font apparaître une évolution de l'ancrage avec le taux de couverture jusqu'à environ $20 \AA$.

\section{Face (100) préparée par nettoyage chimique.}

4.1 ETUDE DES SURFACES. - Le travail de sortie des surfaces (100) préparées par nettoyage chimique est très uniforme (voir Fig. 1). L'écart entre le travail de sortie du type $\mathrm{n}$ et du type $\mathrm{p}$ est faible $\phi_{\mathrm{p}}-\phi_{\mathrm{n}} \approx 50 \mathrm{meV}$. En admettant invariant le travail de sortie de l'électrode de référence (cette invariance a pu être vérifiée à de nombreuses reprises grâce aux surfaces reproductibles que sont les surfaces clivées) on peut comparer leur travail de sortie à ceux des surfaces clivées en bande plate. On trouve $\phi_{\mathrm{p}}-\phi_{0 \mathrm{p}}=-1100 \pm 50 \mathrm{meV}$ et $\phi_{\mathrm{n}}-\phi_{0 \mathrm{n}}=-150 \pm 50 \mathrm{meV}$. Le photovoltage est lui aussi uniforme. Il vaut, à saturation, $350 \mathrm{mV}$ environ pour le type $p$ et $-70 \mathrm{mV}$ environ pour le type $n$. Ces valeurs du photovoltage, exploitées comme précédemment en utilisant les résultats des surfaces clivées, conduisent à $V_{\mathrm{Sn}}=-250 \pm 50 \mathrm{mV}$ et $V_{\mathrm{Sp}}=700 \pm 50 \mathrm{mV}$. L'affinité électronique des surfaces (100) chimiques est inférieure à celle des surfaces (110) clivées. En effet, la différence $\phi_{n}-\phi_{0 n}$ est négative et donc, même si le type $\mathrm{n}$ était en bande plate, on aurait $\chi_{\text {clivé }}-\chi_{\text {chim. }} \approx 150 \mathrm{meV}$ (les dopages des échantillons clivés et préparés par voie chimique sont comparables). Compte tenu du photovoltage et de son signe, il est clair que cette différence est encore plus grande. En utilisant la valeur estimée de $V_{\mathrm{Sn}}$ ou $V_{\mathrm{sp}}$, on aboutit à $\chi_{\text {clivé }}-\chi_{\text {chim. }} \approx 400 \mathrm{meV}$.

En conclusion, les surfaces préparées par voie chimique présentent des états de surface donneurs et accepteurs en densité suffisante pour produire un ancrage du niveau de Fermi en surface à un niveau énergétique situé, dans le cas de la solution utilisée, à environ $400 \mathrm{meV}$ en dessous de la $\mathrm{BC}$. La position de l'ancrage est encore une fois située dans le gap dans la même plage d'énergie que pour les défauts de clivage et qu'après dépôt de métal, du moins pour l'or et l'argent.

Pourtant la composition de ces surfaces est sensiblement différente de celle des surfaces clivées comme le montrent les spectres Auger de la figure 3. Elles comportent de l'oxyde résiduel et du carbone, en quantité relativement faible, mais surtout elles sont pauvres en phosphore. En effet le rapport $\frac{\operatorname{In}(404)}{\mathbf{P}(120)}$ passe de 0,9 pour les surfaces clivées qui comportent le même nombre d'atomes d'indium et de phosphore à 1,8 pour les surfaces chimiques qui, par conséquent, sont riches en indium.

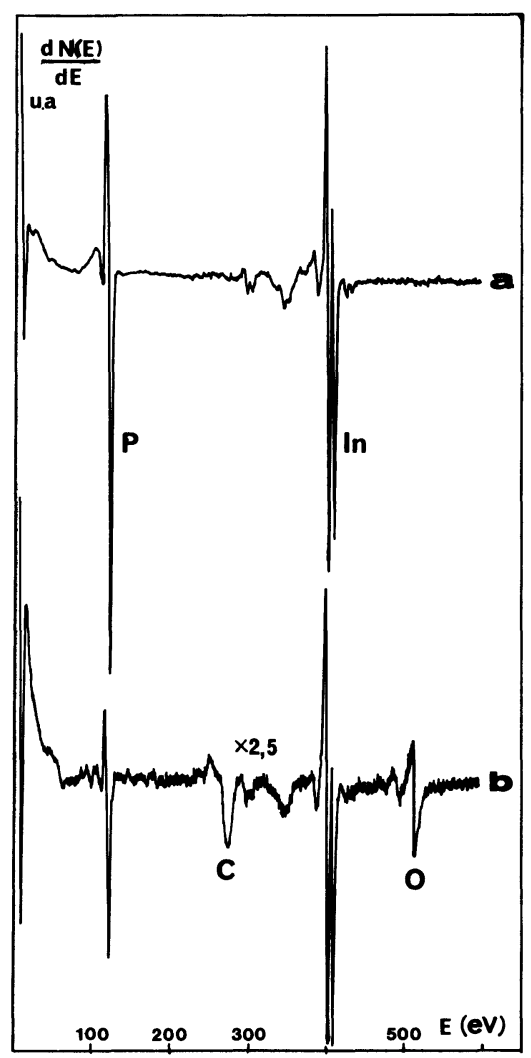

Fig. 3. - Spectres Auger de InP : a - Face (110) clivée. b - Face (100) nettoyée par une solution $3 \mathrm{H}_{2} \mathrm{SO}_{4}, 1 \mathrm{H}_{2} \mathrm{O}_{2}$, $1 \mathrm{H}_{2} \mathrm{O}$.

[InP AES spectrum for : a - (110) cleaved face. $\mathrm{b}-(100)$ face etched by $3 \mathrm{H}_{2} \mathrm{SO}_{4}, 1 \mathrm{H}_{2} \mathrm{O}_{2}, 1 \mathrm{H}_{2} \mathrm{O}$.] 
4.2 ETUDE DES DIODES. - Les caractéristiques des diodes obtenues sont données dans le tableau V. Il apparaît que, par rapport aux surfaces clivées, l'éventail des hauteurs de barrières est resserré autour de la valeur que l'on peut déduire de l'ancrage initial du niveau de Fermi en surface, que l'écart entre $\phi_{B}$ et $\phi_{\mathrm{BC}}$ est plus faible, mais que les tendances propres à la nature du métal sont conservées. Par exemple, la barrière avec l'aluminium sur type $p$ est nettement plus élevée; par exemple, le facteur d'idéalité pour les diodes or/type $\mathrm{p}$ est encore grand. La présence d'une couche d'oxyde résiduel et/ou le fait que le niveau de Fermi soit ancré avant le dépôt ont sensiblement modéré les conséquences sur la barrière des caractéristiques propres à chacune des interactions métal-semiconducteur, le meilleur accord entre les valeurs de $\phi_{\mathrm{B}}$ et de $\phi_{\mathrm{BC}}$ implique une forme de barrière plus proche de la forme parabolique idéale et laisse présager une interface plus abrupte.

\section{Discussion.}

Les résultats que nous avons obtenus sur surfaces clivées avec défauts ou sur surfaces chimiques montrent qu'il existe sur ces surfaces des états situés dans la bande interdite et dont la présence a pour conséquence l'apparition d'une courbure des bandes, dans le sens de l'appauvrissement ou l'inversion, aussi bien pour le type $n$ que pour le type $\mathrm{p}$. Il est donc indispensable que parmi ces états, certains soient donneurs et d'autres accepteurs. Ces états ancrent, pour les surfaces chimiques, ou tendent à ancrer, pour les surfaces clivées, le niveau de Fermi à un niveau d'énergie qui, à la surface, est situé approximativement à $400 \mathrm{meV}$ en dessous de la bande de conduction. Pourtant les origines a priori prévisibles pour ces états sont nettement différentes. D'abord les orientations

Tableau V. - Hauteurs de barrières et facteurs d'idéalité des diodes réalisées sur surface (100) nettoyées chimiquement.

[Barrier hight and ideality factor for the diodes achieved on etched (100) surfaces.]

\begin{tabular}{|c|c|c|c|c|}
\cline { 2 - 5 } \multicolumn{1}{c|}{} & $\mathrm{Ag}$ & $\mathrm{Au}$ & $\mathrm{Al}$ \\
\hline \multirow{4}{*}{ type p } & $\phi_{\mathrm{Bp}}(\mathrm{meV})$ & 880 & 780 & 990 \\
\cline { 2 - 5 } & $n$ & 1,05 & 1,33 & 1,08 \\
\cline { 2 - 5 } & $V_{\mathrm{D}}(\mathrm{mV})$ & 690 & 670 & 870 \\
\cline { 2 - 5 } & $\phi_{\mathrm{Bc}}(\mathrm{meV})$ & 920 & 900 & 1100 \\
\hline \multirow{3}{*}{ type n } & $\phi_{\mathrm{Bn}}(\mathrm{meV})$ & 405 & 470 & 370 \\
\cline { 2 - 5 } & $n$ & 1,05 & 1,02 & 1,07 \\
\hline
\end{tabular}

cristallographiques des faces concernées sont différentes, ensuite dans un cas il s'agit de surfaces parfaitement propres et stœchiométriques avec seulement quelques liaisons pendantes ou perturbées et dans l'autre cas, il s'agit de surface présentant des résidus d'oxydation, des atomes étrangers et un important défaut de stœechiométrie. Il faut donc rechercher à ces états une origine plus intrinsèque au matériau semiconducteur lui-même mais qui ne fasse pas intervenir directement l'arrangement initial des atomes de surface. Le modèle de Spicer et al. [14-16] assorti de l'hypothèse que les défauts puissent être répartis sur quelques couches atomiques, nous paraît le plus approprié. En effet, les états correspondant à ces défauts ne seraient plus à proprement parler des états de surface et donc ne seraient pas sensibles à l'orientation cristallographique. Les différences proprement superficielles se traduiraient alors par des affinités électroniques différentes, ce que nous avons observé.

A l'évidence ce modèle ne peut pas être utilisé sans précaution dans le cas de l'interaction du semiconducteur avec un métal. En effet, il existe maintenant des preuves expérimentales qu'une interdiffusion entre le métal et le semiconducteur se produit à l'interface et que cette interdiffusion dépend beaucoup de la nature du métal. Brillson et al. [31-33] ont montré que l'interface InP-aluminium, métal réactif, est plus abrupte que l'interface InP-or ou argent, métaux non réactifs. Cette notion de réactivité du métal est bien mise en évidence par Williams et al. [27-30, 45] qui montrent que les variations de $\phi_{\mathrm{B}}$ sont corrélées à la chaleur de formation de $\Delta H_{\mathrm{R}}$ à l'interface. Ces différents auteurs ont montré que la diffusion du phosphore dans le métal est réduite si ce dernier est réactif. Nos propres résultats vont dans le sens de ces observations. La diffusion du phosphore est beaucoup moins importante que celle de l'indium que l'on retrouve à la surface d'une couche de $5000 \AA$ d'aluminium. Pour expliquer les variations de la position de l'ancrage du niveau de Fermi avec la nature du métal, variations qui restent cependant limitées dans une plage propre au semiconducteur, on peut envisager deux hypothèses différentes. La première est d'envisager la création d'états nouveaux, caractéristiques du couple métal semiconducteur et donc susceptibles de modifier l'équilibre des charges et donc le niveau de Fermi. La seconde este considérer que suivant la réactivité du métal un ou plusieurs états associés à un ou plusieurs types de défauts est ou sont favorisés par rapport à un ou plusieurs autres. La figure 4 illustre cette possibilité dans le cas d'un donneur et d'un accepteur discrets dont le rapport des densités est constant. On peut voir qu'il suffit de modifier d'un facteur 2 à 3 le rapport de leurs densités pour que l'ancrage se produise au voisinage d'un état ou de l'autre. Cet exemple peut s'appliquer à InP si l'on associe l'état donneur à des lacunes d'In et l'état accepteur à des lacunes de phosphore $[14,16]$. Dans le cas de l'aluminium, où la diffusion du phosphore est bloquée 


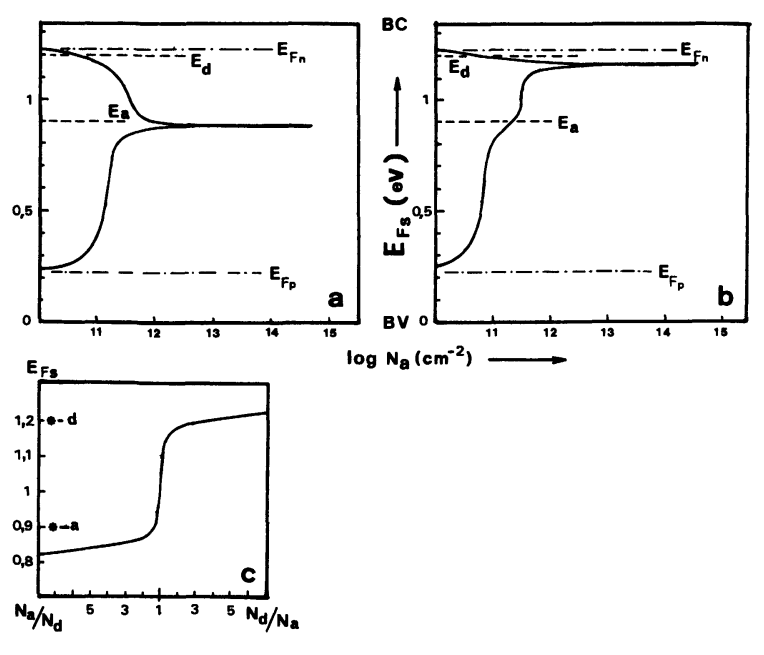

Fig. 4. - Ancrage du niveau de Fermi en surface $E_{\mathrm{Fs}}$ par deux états, donneur et accepteur, discrets d'énergie $E_{\mathrm{a}}=0,9 \mathrm{eV}$ et $E_{\mathrm{d}}=1,2 \mathrm{eV}$ par rapport à la $\mathrm{BV}$. a - Variation du $E_{\mathrm{Fsn}}$ et $E_{\mathrm{Fsp}}$ en fonction de la densité des états $\mathbf{N}$ pour $N_{\mathrm{a}}=1,5 N_{\mathrm{d}} \cdot \mathrm{b}-$ Idem pour $N_{\mathrm{d}}=1,5 N_{\mathrm{a}} \cdot \mathrm{c}-$ Position du niveau de Fermi en surface en fonction du rapport $N_{\mathrm{a}} / N_{\mathrm{d}}$ lorsque les densités d'états sont assez grandes pour que $E_{\mathrm{Fsn}} \approx E_{\mathrm{Fsp}}$.

[Surface Fermi level $\left(E_{\mathrm{Fs}}\right)$ pinning due to two discrete surface states. One is an acceptor with an energy level $E_{\mathrm{a}}=0.9 \mathrm{eV}$ and the other is a donor with $E_{\mathrm{d}}=1.2 \mathrm{eV}$. All level with respect to the VBM. a $-E_{\mathrm{Fsn}}$ and $E_{\mathrm{Fsp}}$ versus density of the states $N$ for $N_{\mathrm{a}}=1.5 N_{\mathrm{d}}$. b - For $N_{\mathrm{d}}=1,5 N_{\mathrm{a}}$. $\mathrm{c}-E_{\mathrm{Fs}}$ versus $N_{\mathrm{a}} / N_{\mathrm{d}}$ when state densities are high enough to produce $E_{\mathrm{Fsn}} \approx E_{\mathrm{Fsp}}$.]

par des liaisons avec le métal et où la diffusion de l'indium reste importante, une densité du donneur supérieure à celle de l'accepteur produirait un ancrage proche de la BC. Des calculs récents effectués par Daw et Smith [46, 47] et par Allen et Dow $[48,49]$ ont montré que les niveaux associés aux défauts, lacunes mais aussi défauts d'antisite, étaient situés dans la bande interdite à des énergies pouvant rendre compte des ancrages du niveau de Fermi observés. Même si des controverses existent sur le niveau énergétique associé à chaque type de défaut, ces calculs apportent un support théorique à une explication de l'ancrage par des imperfections intrinsèques au semiconducteur. Une modulation de la proportion des différents défauts par la réactivité du métal semble une explication satisfaisante aux déplacements limités du niveau de Fermi à l'interface. Elle s'accorde bien avec le fait expérimental que sur les surfaces chimiques, où ces défauts préexistent très certainement, les variations de $\phi_{\mathrm{B}}$ sont plus faibles et à peu près centrées sur la position initiale de l'ancrage. En effet, la présence d'oxyde résiduel et la satisfaction des liaisons du semiconducteur qu'elle implique, limite l'action du métal. L'hypothèse d'états caractéristiques de liaisons métalIn et métal-P a contre elle le fait que pratiquement deux positions de l'ancrage seulement sont observées pour une large variété de métaux déposés sur surfaces clivées ce qui veut bien dire qu'à deux métaux différents peut correspondre la même barrière.

Le modèle de Freeouf et al. [23-25] s'accommode mal de la présence d'états et singulièrement d'un effet non négligeable d'états préexistants. En effet, il implique que les états n'existent pas, car s'ils existaient ils écranteraient le travail de sortie "effectif» de la même façon qu'ils doivent écranter celui du métal pour imposer la position du niveau de Fermi.

Le modèle de Sebenne et al. [26] attribue intrinsèquement les états au semiconducteur puisqu'il considère que la satisfaction des liaisons de ce dernier ramène dans la bande interdite les états qui avaient été repoussés hors des bandes par la relaxation des atomes de surface. Il explique ainsi que chaque semiconducteur ait un comportement propre. Mais ce modèle qui considère une interface plutôt abrupte devra s'accommoder des phénomènes d'interdiffusion observés, des variations de l'ancrage avec la réactivité et d'une influence non négligeable de la surface initiale.

\section{Conclusion.}

Nous avons étudié dans cet article la formation de l'interface et du contact Schottky lors du dépôt d'or, d'argent ou d'aluminium sur les surfaces (110) et (100) de InP obtenues respectivement par clivage et par nettoyage chimique. Il ressort de nos résultats que, dans tous les cas observés autres que celui des surfaces (110) parfaitement clivées, le niveau de Fermi est ancré à la surface ou à l'interface métal-InP à un niveau situé à l'intérieur d'une plage d'énergie relativement étroite comprise approximativement entre 0,2 et $0,5 \mathrm{eV}$ en dessous de la bande de conduction, en accord satisfaisant avec les observations publiées par d'autres auteurs.

Dans le cas des surfaces parfaitement clivées, l'origine de l'ancrage est dans l'interaction métalsemiconducteur et la nature du métal influe sur la position de l'ancrage. Mais le même ancrage peut être produit par d'autres causes. C'est le cas des surfaces (100) chimiques ou (110) présentant des défauts de clivage. L'explication de l'ancrage apparaît donc comme liée au matériau lui-même ou plus précisément à une modification du matériau localisée à la surface ou à l'interface. Aussi le modèle des défauts de Spicer et al., nous paraît le plus approprié. Nous pensons que les différentes interactions se produisant avec la surface aboutissent à la création, dans les premières couches du semiconducteur, d'un certain nombre de défauts. Ces défauts, vraisemblablement lacunes de In ou de $\mathbf{P}$ mais peut-être aussi défauts plus complexes, produisent l'ancrage du niveau de Fermi car ils sont associés à des états électroniques accepteurs et donneurs situés dans la bande interdite. Pour expliquer les fluctuations du niveau où se produit l'ancrage, on peut envisager des variations dans la proportion relative de la densité des états donneurs et accepteurs, variations liées aux détails de la formation de l'inter- 
face et en particulier aux phénomènes d'interdiffusion modulés par la réactivité du métal vis-à-vis des deux constituants du semiconducteur ou par la présence d'une couche intermédiaire telle la couche d'oxyde. Par exemple, l'ancrage proche de la bande de conduction observé dans le cas de l'aluminium peut s'expliquer, dans le cadre d'un modèle simple à deux états discrets, par une limitation de la diffusion des atomes de phosphore du fait de leur liaison avec les atomes d'aluminium. La densité des états donneurs, liée aux lacunes d'indium, serait supérieure à celle des états accepteurs liés aux lacunes de phosphore.

C'est l'hypothèse que les états sont répartis au moins sur quelques couches atomiques qui peut, à notre avis, le mieux rendre compte de l'ensemble des observations expérimentales. D'une part, la couche d'états peut avoir une longueur de Debye équivalente inférieure à sa propre épaisseur et de ce fait on comprend qu'elle puisse écranter l'effet soit du travail de sortie du métal ou de toute couche composite intermédiaire, soit des modifications de l'affinité électronique mises en évidence expérimentalement. D'autre part, la couche d'état peut correspondre à une déformation du sommet de la barrière par rapport à la forme parabolique théorique et expliquer ainsi le désaccord entre les mesures de $\phi_{\mathrm{B}}$ effectuées par $I(V)$ et par $C(V)$ et certaines valeurs élevées du facteur d'idéalité.
[1] Motr, N. F., Proc. Cambridge Philos. Soc. 34 (1938) 568.

[2] Sснотткy, W., Naturwiss 26 (1938) 843.

[3] Schotтky, W., Z. Phys. 113 (1939) 367.

[4] Bethe, H. A., MIT Radiation lab. Rep. 43-12 (1942).

[5] Bardeen, J., Phys. Rev. 71 (1947) 717.

[6] Cowley, A. M. and Sze, S. M., J. Appl. Phys. 36 (1965) 3212.

[7] Voir par exemple RHODERICK, E. H., Métal semiconductor contacts. Monographs in Electrical and Electronic Engineering (Clarendon Press, Oxford) 1980.

[8] Heine, V., Phys. Rev. A 138 (1965) 1689.

[9] Bennett, A. J. and Duke, C. B., Phys. Rev. 162 (1967) 578.

[10] Inkson, J. C., J. Phys. C 6 (1973) 1350.

[11] Zhang, H. I. and Schluter, M., J. Vac. Sci. Technol. 15 (1978) 1384.

[12] Chelikowsky, J. R. and Cohen, M. L., Phys. Rev. B 20 (1979) 4150.

[13] Voir par exemple l'article de revue de BRILlson, L. J., Surface Science Reports 2 № 2 (1982).

[14] Spicer, W. E., Chye, P. W., Skeath, P. R., Su, C. Y. and Lindau, I., J. Vac. Sci. Technol. 16 (1979) 1422.

[15] Spicer, W. E., Lindau, I., Skeath, P., Su, C. Y. and Chye, P., Phys. Rev. Lett. 44 (1980) 420.

[16] Spicer, W. E., Lindau, I., SKeath, P. and Su, C. Y., J. Vac. Sci. Technol. 17 (1980) 1019.

[17] Williams, R. H., Varma, R. R. and Montgomery, V., J. Vac. Sci. Technol. 16 (1979) 1418.

[18] Williams, R. H., J. Vac. Sci. Technol. 18 (1981) 929.

[19] Brillson, L. J., J. Vac. Sci. Technol. 15 (1978) 1378.

[20] Brillson, L. J., Margaritondo, G., Stoffel, N. G., BAUER, R. S., BACHRACH, R. Z. and HaNSSON, G., J. Vac. Sci. Technol. 17 (1980) 880.

[21] Brillson, L. J., Bauer, R. S., Bachrach, R. Z. and Hansson, G., Phys. Rev. B 23 (1981) 6204.

[22] Brucker, C. F., Brillson, L. J., Katnani, A. D., Stoffel, N. G. and Margaritondo, G., J. Vac. Sci. Technol. 21 (1982) 590.
[23] Freeouf, J. and Woodall, J. M., Appl. Phys. Lett. 39 (1981) 727.

[24] Woodall, J. M. and Freeouf, J., J. Vac. Sci. Technol. 19 (1981) 794.

[25] Woodall, J. M. and Freeouf, J., J. Vac. Sci. Technol. 21 (1982) 574.

[26] Bolmont, D., Chen, P., Proix, F. and Sebenne, C. A., J. Phys. C 15 (1982) 3639.

[27] McKinley, A., Parke, A. W. and Williams, R. H., J. Phys. C 13 (1980) 6723.

[28] McKinley, A., Hughes, G. J. and Williams, R. H., J. Phys. C 15 (1982) 7049.

[29] Montgomery, J. V. and Williams, R. H., J. Phys. C 15 (1982) 5887.

[30] Williams, R. H., McKinley, A., Hughes, G. J., MontGOMERY, V. and McGovern, I. T., J. Vac. Sci. Technol. 21 (1982) 594.

[31] Brillson, L. J., Brucker, C. F., Katnani, A. D., Stoffel, N. G. and Margaritondo, G., Appl. Phys. Lett. 38 (1981) 784.

[32] Brillson, L. J., Brucker, C. F., Katnani, A. D., Stoffel, N. G. and Margaritondo, G., J. Vac. Sci. Technol. 19 (1981) 661.

[33] Brillson, L. J., Brucker, C. F., Katnani, A. D., Stoffel, N. G., Daniels, R. and MARGaritondo, G., J. Vac. Sci. Technol. 21 (1982) 564.

[34] Chye, P. W., Lindau, I., Pianetta, P., Garner, C. M., Su, C. Y. and SPICER, W. E., Phys. Rev. B 18 (1978) 5545.

[35] Babalola, I. A., Petro, W. G., Kendelewicz, T., LINDAU, I. and SPICER, W. E., J. Vac. Sci. Technol. A 1 (1983) 762.

[36] Kendelewicz, T., Petro, W. G., Babalola, I. A., Silberman, J. A., Lindau, I. and Spicer, W. E., Phys. Rev. B 27 (1983) 3366.

[37] Palau, J. M., Testemale, E. and Lassabatère, L., J. Vac. Sci. Technol. 19 (1981) 192.

[38]. Palau, J. M., Testemale, E., Ismail, A. and LassabaTÈRE, L., Solid State Elec. 25 (1982) 285.

[39] Brillson, L. J., J. Vac. Sci. Tecnol. 16 (1979) 1137. 
[40] Brillson, L. J., Thin Solid Films 89 (1982) L 27 et Eglash, S., Spicer, W. E. and Lindau, I., Thin Solid Films 89 (1982) L 35.

[41] Palau, J. M., Testemale, E., Ismail, A. and LassabaTÈre, L., J. Vac. Sci. Technol. 21 (1982) 6.

[42] Palau, J. M., Ismail, A., Testemale, E. and LassabaTÈrE, L., Proceedings of the 16th Inter. Conf. On Phys. of Semiconductor Montpellier, II 860 (1982).

[43] Palau, J. M., Pouvil, P., Testemale, E. et LassabaTÈRE, L., le Vide, les Couches Minces 198 (1979) 301.

[44] Testemale, E., Palau, J. M., Pouvil, P., Lassabatère,
L. and Legoascoz, V., Thin Solid Films 71 (1980) 161.

[45] Williams, R. H., Montgomery, V. and Varma, R. R., J. Phys. C 11 (1978) L 735.

[46] Daw, M. S. and Smith, D. L., J. Vac. Sci. Technol. 17 (1980) 1028.

[47] Daw, M. S. and Smith, D. L., Appl. Phys. Lett. 36 (1980) 690.

[48] Allen, R. E. and Dow, J. D., J. Vac. Sci. Technol. 19 (1981) 383

[49] Dow, J. D. and Allen, R. E., J. Vac. Sci. Technol. 20 (1982) 65 . 